\title{
MYCN mediates TFRC-dependent ferroptosis and reveals vulnerabilities in neuroblastoma
}

\author{
Yuxiong $\mathrm{Lu}^{1,2}$, Qing Yang ${ }^{3}$, Yubin Su${ }^{4}$, Yin $\mathrm{Ji}^{5}$, Guobang Li ${ }^{3}$, Xianzhi Yang ${ }^{3}$, Liyan $\mathrm{Xu}^{3}$, Zhaoliang $\mathrm{Lu}^{1}$, Jiajun Dong ${ }^{6}$, \\ Yi Wu', Jin-Xin Bei $\mathbb{D}^{2,7}$, Chaoyun Pan ${ }^{3,8}$, Xiaoqiong Gu${ }^{1}$ and Bo Li $\mathbb{C}^{2,3,7,8}$
}

\begin{abstract}
MYCN amplification is tightly associated with the poor prognosis of pediatric neuroblastoma (NB). The regulation of NB cell death by MYCN represents an important aspect, as it directly contributes to tumor progression and therapeutic resistance. However, the relationship between MYCN and cell death remains elusive. Ferroptosis is a newly identified cell death mode featured by lipid peroxide accumulation that can be attenuated by GPX4, yet whether and how MYCN regulates ferroptosis are not fully understood. Here, we report that MYCN-amplified NB cells are sensitive to GPX4-targeting ferroptosis inducers. Mechanically, MYCN expression reprograms the cellular iron metabolism by upregulating the expression of TFRC, which encodes transferrin receptor 1 as a key iron transporter on the cell membrane. Further, the increased iron uptake promotes the accumulation of labile iron pool, leading to enhanced lipid peroxide production. Consistently, TFRC overexpression in NB cells also induces selective sensitivity to GPX4 inhibition and ferroptosis. Moreover, we found that MYCN fails to alter the general lipid metabolism and the amount of cystine imported by System $X_{c}(-)$ for glutathione synthesis, both of which contribute to ferroptosis in alternative contexts. In conclusion, NB cells harboring MYCN amplification are prone to undergo ferroptosis conferred by TFRC upregulation, suggesting that GPX4-targeting ferroptosis inducers or TFRC agonists can be potential strategies in treating MYCN-amplified NB.
\end{abstract}

\section{Introduction}

Neuroblastoma (NB) is the most common extracranial solid tumor in human infants, with $90 \%$ of cases diagnosed by the age of $5^{1}$. Genetically, frequent amplifications of the oncogene $M Y C N$ are identified in patients with $\mathrm{NB}$, predicting poor prognosis independent of other factors $^{2}$. Although $M Y C N$ inhibition leads to the suppression of NB in vitro, there are technical challenges in targeting $M Y C N$ clinically ${ }^{3,4}$. MYCN orchestrates multiple molecular pathways for cell growth, survival, metabolism,

\footnotetext{
Correspondence: Xiaoqiong Gu (guxiaoqiong@gwcmc.org) or

Bo Li (libo47@mail.sysu.edu.cn)

${ }^{1}$ Clinical Biological Resource Bank, Guangzhou Institute of Pediatrics,

Guangzhou Women and Children's Hospital, Zhongshan School of Medicine,

Sun Yat-sen University, Guangzhou, China

${ }^{2}$ State Key Laboratory of Oncology in South China, Collaborative Innovation

Center for Cancer Medicine, Sun Yat-sen University Cancer Center, Guangzhou, China

Full list of author information is available at the end of the article

These authors contributed equally: Yuxiong Lu, Qing Yang, Yubin Su, Yin Ji

Edited by B. Zhivotovsky
}

and death, dictating cancer cell fate and tumor progres$\operatorname{sion}^{5,6}$. In order to explore new treatments against $M Y C N$-amplified $\mathrm{NB}$, it is necessary to gain a deeper understanding of the biological functions mediated by $M Y C N$.

Among the key cellular processes governed by $M Y C N$, cell death is a puzzling phenomenon. On one hand, it is the cause of tumor growth under nutrient-deprived conditions whose adaptation promotes NB progression ${ }^{7,8}$; on other hand, abnormal expression of $M Y C N$ leads to the direct activation of cell death pathways ${ }^{9,10}$. This contradiction indicates that $M Y C N$ finely regulates the balance between cell survival and cell death. Recently, researchers discovered a new type of programmed cell death, namely ferroptosis, which is characterized by intracellular iron disorder and accumulation of lipid peroxides in the cell membrane $^{11}$. Cell culture and animal experiments have shown that induction of ferroptosis can eliminate selective tumor cells that are resistant to apoptosis, such as in

\section{(c) The Author(s) 2021}

\footnotetext{
(c) Open Access This article is licensed under a Creative Commons Attribution 4.0 International License, which permits use, sharing, adaptation, distribution and reproduction cc) in any medium or format, as long as you give appropriate credit to the original author(s) and the source, provide a link to the Creative Commons license, and indicate if changes were made. The images or other third party material in this article are included in the article's Creative Commons license, unless indicated otherwise in a credit line to the material. If material is not included in the article's Creative Commons license and your intended use is not permitted by statutory regulation or exceeds the permitted use, you will need to obtain permission directly from the copyright holder. To view a copy of this license, visit http://creativecommons.org/licenses/by/4.0/.
} 
kidney cancer ${ }^{12}$, breast cancer ${ }^{13}$, lung cancer $^{14}$, etc., which provides a new venue for overcoming drug resistance. System $\mathrm{X}_{\mathrm{c}}(-)$ and GPX4 inhibition are the most common methods to induce ferroptosis. System $X_{c}(-)$ imports cystine as a key substrate for synthesizing glutathione (GSH), which is the reducing equivalent used by GPX4 to antagonize ferroptosis ${ }^{15}$. Although $M Y C N$ has a role in regulating cellular redox balance ${ }^{16}$, the potential effects of $M Y C N$ on ferroptosis remain unclear.

In this study, we identified a new regulatory mechanism of ferroptosis by MYCN. We found that NB cells overexpressing $M Y C N$ are particularly sensitive to ferroptosis induced by GPX4 inhibition rather than system $\mathrm{X}_{\mathrm{c}}(-)$ blockage. Moreover, MYCN amplification fails to cause significant changes in lipid species, suggesting that alternations of iron metabolism are mainly accounting for $M Y C N$ regulated ferroptosis. Therefore, we analyzed the expression of iron-related genes and revealed that TFRC, which encodes transferrin receptor 1 as an essential iron transporter on the cell membrane, plays a key role in the process of $M Y C N$ regulated ferroptosis. Similar to $M Y C N$, TFRC significantly increases the intracellular iron load, and TFRC upregulation confers cell sensitivity to ferroptosis induced by GPX4 inhibition. Considering that $M Y C N$ and TFRC are both deregulated in a variety of tumor types, our finding points to a tumor vulnerability that can be therapeutically exploited.

\section{Results}

\section{MYCN is a regulator of GPX4-dependent ferroptosis}

To study $M Y C N$-dependent cellular processes in NB, we examined a collection of 147 genes essential for the survival of $M Y C N$-amplified NB cells ${ }^{17}$ through pathway analysis using the Molecular Signatures Database v7.2. We discovered that genes regulating cell death are significantly enriched in this collection (Fig. 1A). Furthermore, we performed RNA-seq and gene set enrichment analysis (GSEA) of six human NB cell lines (MYCN amplified SK-N-BE2, BE(2)-C, NLF, SK-N-DZ, and $M Y C N$ non-amplified SHEP, SK-N-AS), and found that MYCN expression is closely associated with levels of ferroptosis-related genes as summarized by Liang et al. ${ }^{18}$ (Fig. 1B and Supplementary Table 1). Next, we compared the ferroptosis-related genes with those regulating MYCN-dependent cell survival (Supplementary Table 2), and identified GPX4 as the only overlapping gene (Fig. 1C). GPX4 is a well-established ferroptosis regulator neutralizing the oxidized lipid species ${ }^{19}$. To confirm the above results, multiple $\mathrm{NB}$ cell lines were treated respectively with two classic ferroptosis inducer, (1S, 3 R)-RSL3 (hereafter RSL3 for short), a highly specific GPX4 inhibitor, and Erastin, a cystine-glutamate antiporter (system $\left.\mathrm{X}_{\mathrm{c}}(-)\right)$ inhibitor. Interestingly, $M Y C N$ amplification significantly increases the sensitivity of $\mathrm{NB}$ cells to RSL3, whereas all NB cells exhibit similar resistance to Erastin (Fig. 1D and Fig. S1A). Consistently, the effects of an alternative system $\mathrm{Xc}(-)$ inhibitor sulfasalazine (SAS) ${ }^{20}$ are weakly associated with $M Y C N$ amplification (Fig. S1B, C). These findings imply that $M Y C N$ is a key regulator of ferroptosis, and GPX4 might play an essential role in this process. Indeed, high expression of GPX4 in NB tumor tissues predicts poor prognosis (Fig. 1E). However, RNA-seq data reveal that there is no significant correlation between GPX4 and MYCN expression (Fig. 1F and Fig. S1D). Therefore, $M Y C N$ regulates GPX4-dependent ferroptosis likely through an unknown mechanism.

\section{MYCN confers cell sensitivity to ferroptosis upon GPX4 inhibition}

To confirm that $M Y C N$ is linked to ferroptosis, we ectopically expressed MYCN in SHEP, a MYCN nonamplified NB cell line, and found that MYCN expression significantly increases lipid peroxide contents. Conversely, lipid peroxides are reduced when $M Y C N$ is depleted in SKN-BE2, a MYCN amplified NB cell line (Fig. 2A, B). Next, MYCN overexpression significantly increases the sensitivity of SHEP cells to RSL3, but not Erastin. Moreover, MYCN induced sensitivity towards RSL3 can only be rescued by treating cells with ferroptosis inhibitors, but not other cell death inhibitors (Fig. 2C). These results are further supported by the data in $M Y C N$ amplified SK-N-BE2 cells with shRNA-mediated MYCN depletion (Fig. 2D).

The function of GPX4 is to convert toxic lipid peroxides to nontoxic alcohol lipids using the reducing equivalent glutathione. The system $\mathrm{X}_{\mathrm{c}}(-)$ imports cystine, the extracellular form of cysteine, for glutathione synthesis, therefore functioning upstream of GPX4. To test whether the intracellular cysteine level can be regulated by $M Y C N$, we use mass spectrometry to identify altered metabolites upon $M Y C N$ overexpression, and found that $M Y C N$ does not significantly affect the intracellular cysteine level (Fig. 2E). Meanwhile, our metabolic analysis confirms that MYCN amplification elevates serine levels, which is consistent with previous findings such as by Xia et al. ${ }^{21}$. These results demonstrate that cellular cystine import is independent of $M Y C N$. Together, we conclude that $M Y C N$ induces NB cell sensitivity to ferroptosis upon GPX4 inhibition, through a mechanism non-correlated with cysteine metabolism.

\section{MYCN increases the intracellular iron load}

Deregulated iron metabolism and abnormal lipid synthesis are both required to induce ferroptosis ${ }^{22}$. To this end, we examined the fatty acid content upon $M Y C N$ overexpression by mass spectrometry and found that MYCN has no significant influence on cellular fatty acid pools (Fig. 3A). Next, we analyzed the expression of 
A

GO terms of biological process for MYCN dependent genes

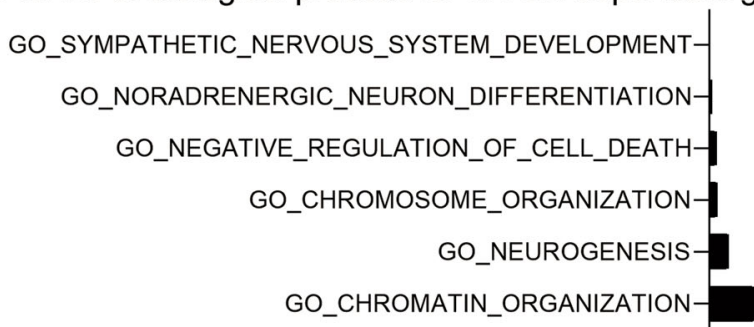

GO_AUTONOMIC_NERVOUS_SYSTEM_DEVELOPMENT-

GO_NEGATI

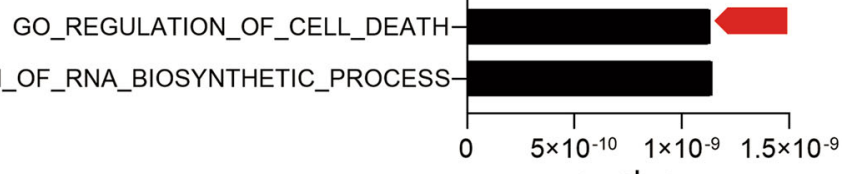

B

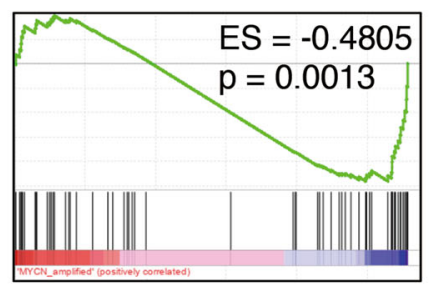

D

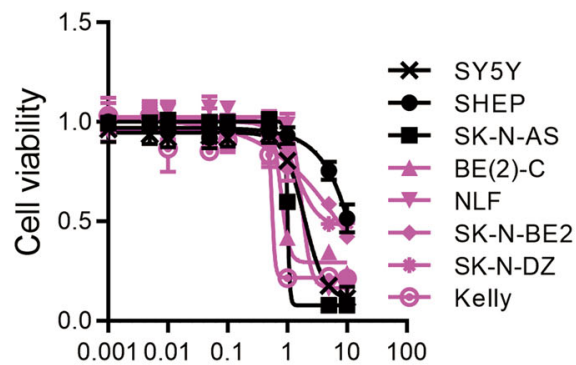

[Erastin], $\mu \mathrm{M}$

E

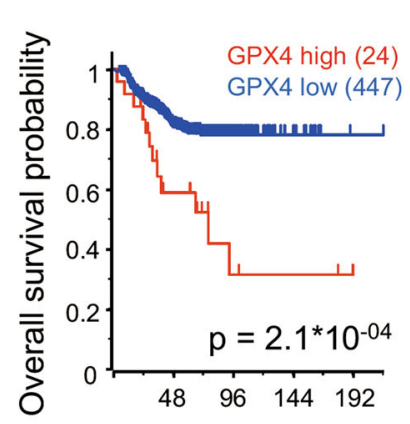

Follow up on months

$\mathbf{F}$
C

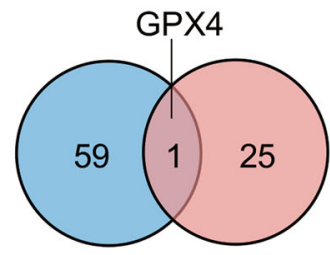

60 ferroptosis related genes

$p$ value

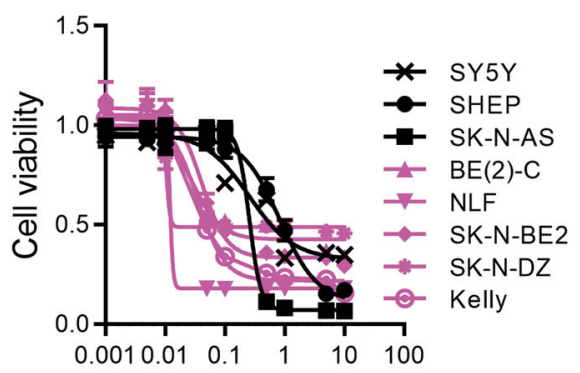

[RSL3], $\mu \mathrm{M}$

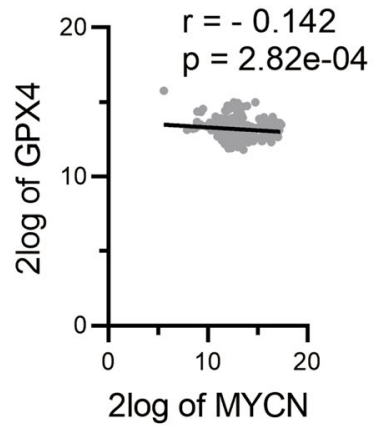

Fig. $1 M Y C N$ is a regulator of GPX4-dependent ferroptosis. A Genes selectively dependent on MYCN for NB cell survival are enriched within a variety of GO terms of biological processes, and the top nine processes are shown according to $p$ values. B GSEA assays of ferroptosis-related genes showing enrichment with MYCN amplification based on RNA-seq data of six human NB cell lines. ES, enrichment score. C GPX4 is shared between ferroptosis-related and MYCN-dependent survival genes. D Cell viabilities when different concentrations of the GPX4 inhibitor (1S, 3R)-RSL3 (RSL3) and system $X_{c}(-)$ inhibitor Erastin were used as ferroptosis inducers in multiple human NB cell lines (MYCN amplified SK-N-BE2, BE(2)-C, NLF, SK-N-DZ, and Kelly are represented in pink; and MYCN non-amplified SHEP, SK-N-AS, and SY5Y are shown in black; $n=4)$. E Kaplan-Meier survival curves for NB patients based on GPX4 mRNA expression. Gene expression and clinical data were retrieved from GSE45547. F Correlation analysis between GPX4 and MYCN expression based on the data retrieved from GSE45547. ${ }^{*} p<0.05,{ }^{* *} p<0.01$. 
A

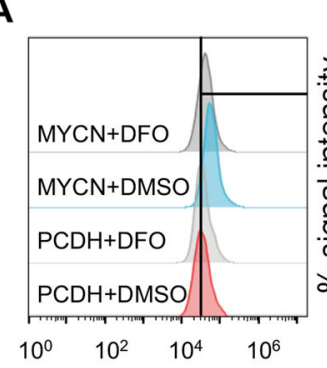

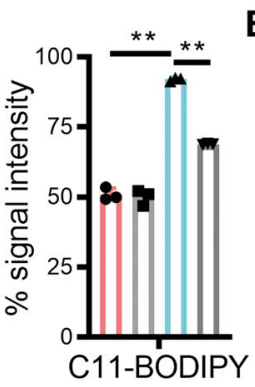
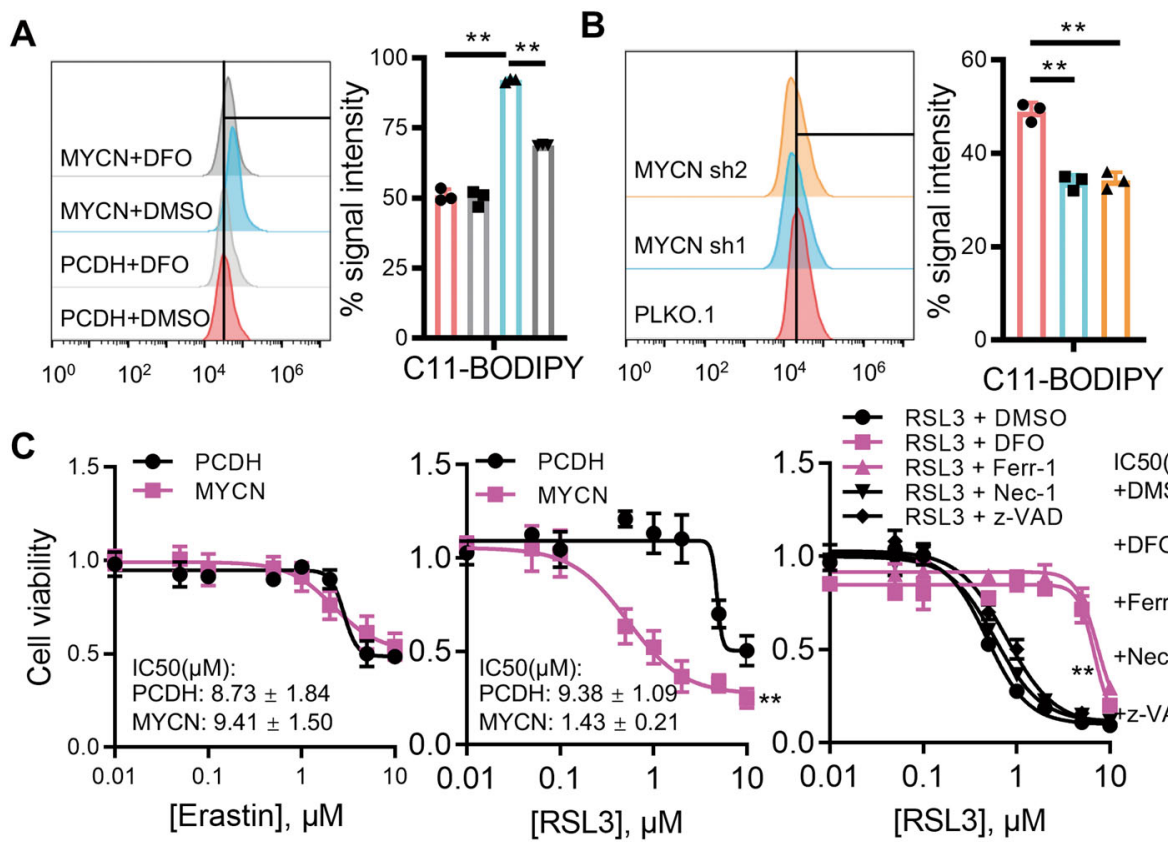

$\because \quad \mathrm{RSL} 3+\mathrm{DMSO}$

- RSL3 + DFO

- RSL3 + Ferr-1 IC50 $(\mu \mathrm{M})$ $\rightarrow$ RSL3 + Nec-1 +DMSO: 0.59 $\curvearrowleft$ RSL3 + z-VAD $\quad \pm 0.04$ +DFO: 5.86 $\pm 0.76$

+Ferr-1: 7.43 $\pm 0.53$

D

E

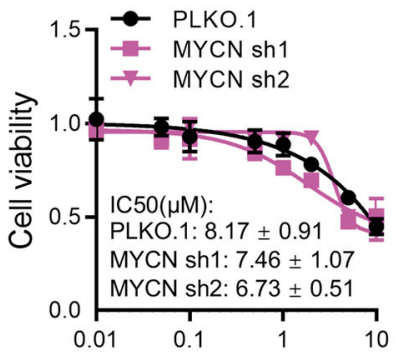

[Erastin], $\mu \mathrm{M}$
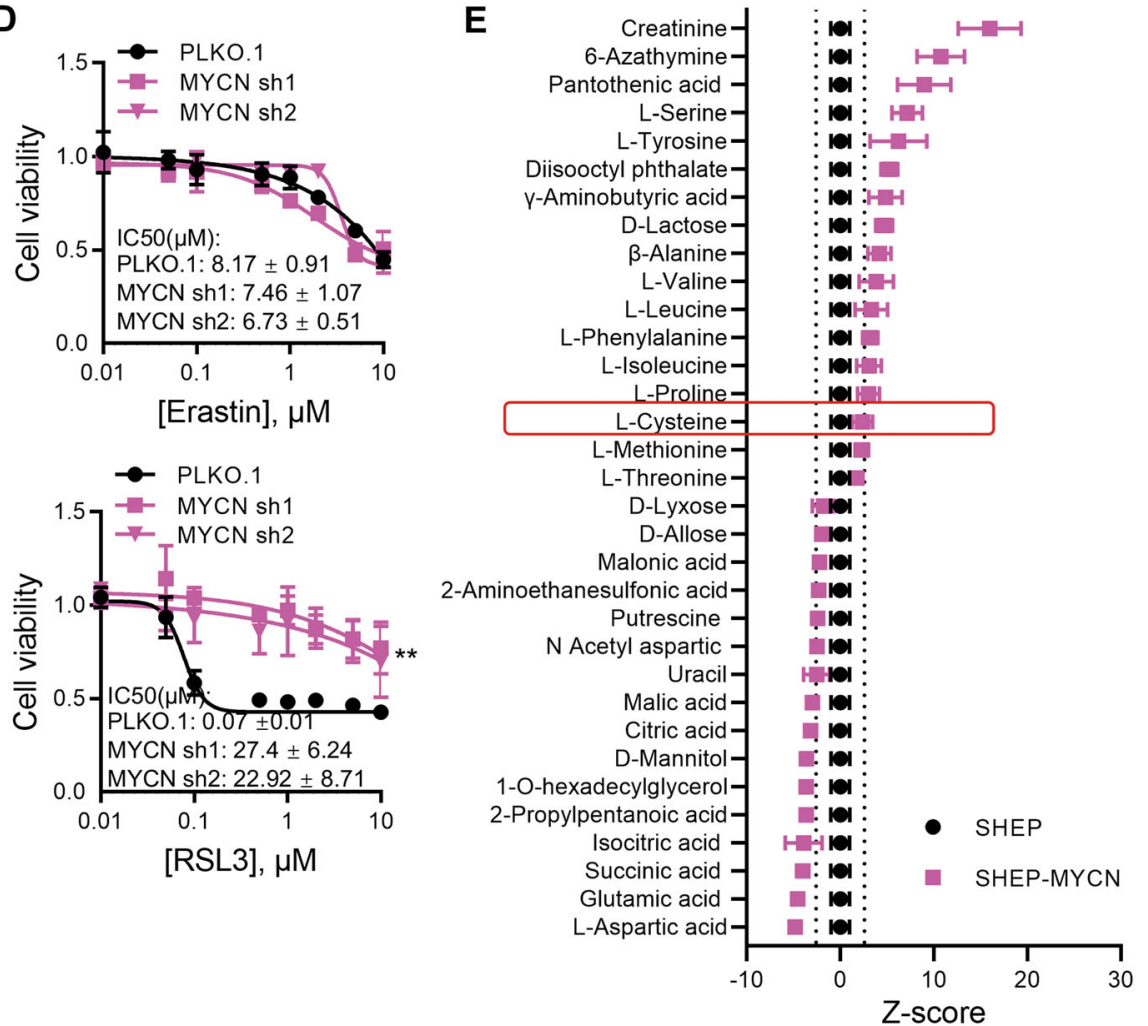

Fig. 2 MYCN confers cell sensitivity to ferroptosis upon GPX4 inhibition. Lipid peroxides were detected by flow cytometry after incubation with C11-BODIPY in SHEP (A) or SK-N-BE2 (B) cells with or without MYCN. DFO, desferrioxamine, $n=3$. C Cell viabilities of SHEP cells with or without MYCN overexpression incubated with Erastin (left), RSL3 (middle), or RSL3 combined with either ferroptosis inhibitor (10 $\mu \mathrm{M}$ DFO, $1 \mu \mathrm{M}$ Ferr-1), apoptosis inhibitor $(20 \mu \mathrm{M} z-V A D)$, or necroptosis inhibitor $(2 \mu \mathrm{M}$ Necrostatin-1) (right). D Cell viabilities of SK-N-BE2 cells with or without MYCN knockdown incubated with Erastin or RSL3. E Metabolic analysis of SHEP cells with or without MYCN. Z-score \pm 2.75 is corresponding to $p=0.01$ and depicted as a dotted line. Data are presented as mean \pm SD of three replicates. Two-tailed unpaired $t$-tests were performed to calculate $p$ values. ${ }^{* *} p<0.01$. 


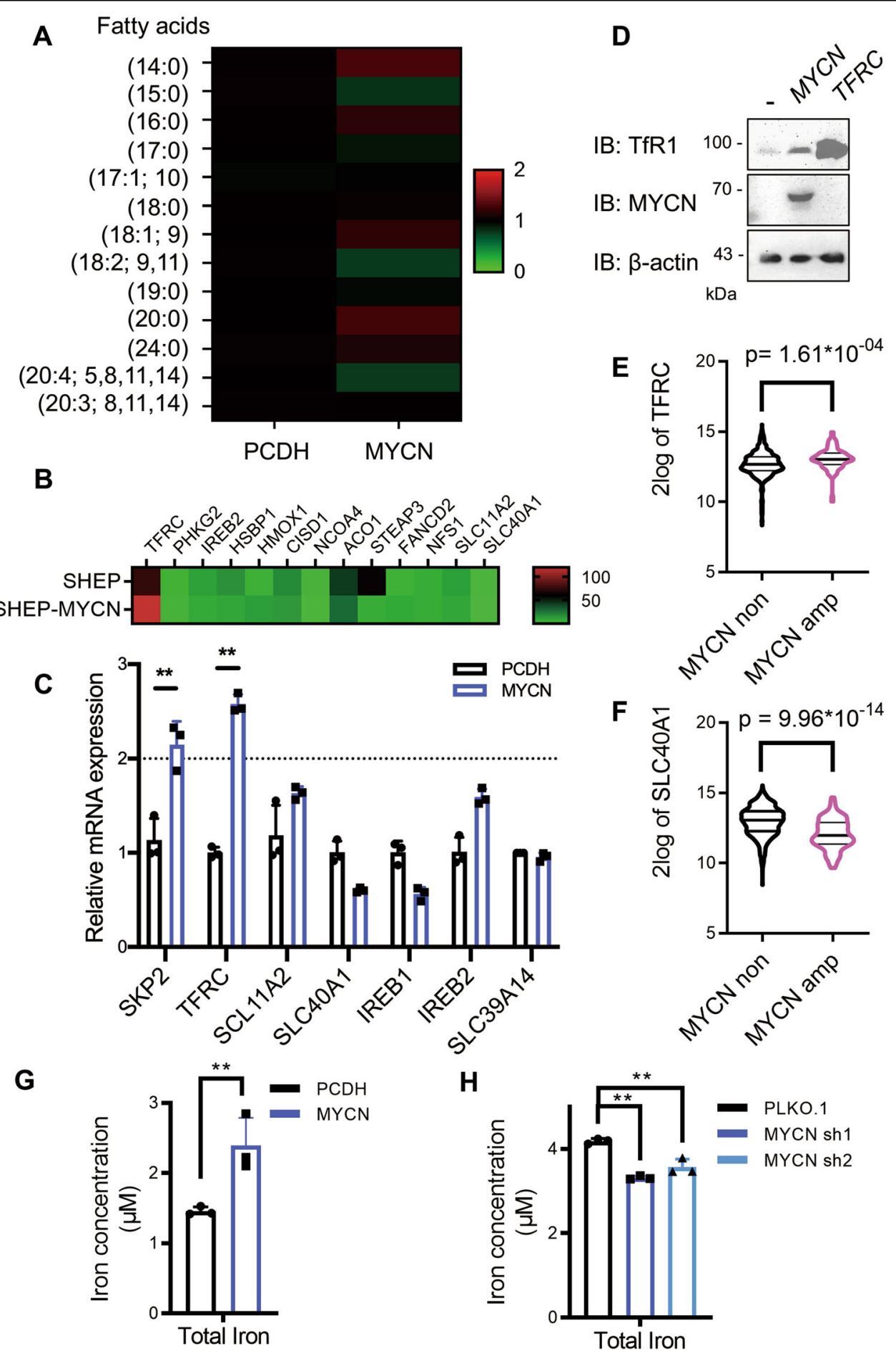

Fig. 3 MYCN increases the intracellular iron load. A Heatmap showing changes in an array of fatty acids in SHEP cells with or without MYCN. Data are shown as relative changes in abundance compared to the PCDH vector. B Heatmap showing the expression profile of ferroptosis-associated metabolic genes. C Expression of MYCN induced iron metabolic genes analyzed by RT-qPCR, and SKP2 is positive control of MYCN targeted genes. D Western blot analysis of SHEP cells with or without MYCN expression using indicated antibodies. E, F TFRC and SLC40A1 expression in NB tumor tissues with or without MYCN amplification. Expression data were retrieved from GSE45547. Cellular iron load is detected with the colorimetric ferrozine-based assay in SHEP G and SK-N-BE2 $\mathbf{H}$ cells with or without MYCN, and values are normalized to protein concentration. Data are presented as mean \pm SD of three replicates. Two-tailed unpaired $t$-tests were performed to calculate $p$ values. ${ }^{* *} p<0.01$. 
several ferroptosis-related genes as summarized by Hassannia et $\mathrm{al}^{23}$, and identified TFRC as the most upregulated one both in RNA-seq data (Fig. 3B) and RT-PCR results (Fig. 3C). TFRC encodes transferrin receptor 1 (TfR1), which is the prevalent iron importer on the cell membrane. Consistently, MYCN upregulation enhances the protein level of TfR1 in NB cells (Fig. 3D), which parallels with TFRC levels in NB tumor tissues (Fig. 3E). Interestingly, we noticed that the expression of SLC4OA1, which encodes the only known iron exporter ferroportin, is slightly but reproductively reduced upon $M Y C N$ overexpression (Fig. 3C), and RNA-seq analysis of NB tumor tissues also supports this argument (Fig. 3F). These results suggest that MYCN tends to increase the iron load in NB cells. To confirm this, we tested the level of the total iron pool (ferric plus ferrous ion) upon $M Y C N$ manipulation. As expected, $M Y C N$ overexpression increases the iron level in SHEP cells, whereas MYCN depletion reduces the iron level in SK-N-BE2 cells (Fig. 3G, H). These results indicate that $M Y C N$ mediates GPX4-dependent ferroptosis likely via regulating iron metabolism.

\section{TFRC photocopies MYCN to increase the labile iron pool}

To confirm that $M Y C N$-induced TFRC expression promotes iron uptake, we overexpressed TFRC in SHEP and depleted TFRC in SK-N-BE2 cells, respectively. Indeed, TFRC expression levels correlate with the cellular iron load (Fig. 4A, B). The $\mathrm{Fe}^{3+}$ ion transported by TfR1 undergoes endocytosis, reduction to $\mathrm{Fe}^{2+}$, and importation to the cytosol composing the labile iron pool (LIP). $\mathrm{Fe}^{2+}$ promotes the formation of lipid peroxides by Fenton reactions. Based on these knowledges, we reasoned that increased cellular iron load would result in LIP upregulation, thereby promoting lipid peroxide formation. To test this, we detected intracellular LIP levels with the calcein acetoxymethyl ester (Cal-AM) probe as previously described $^{24}$. Indeed, the LIP levels are increased upon TFRC overexpression and deceased upon TFRC knockdown (Fig. 4C, D), which photocopy the effects of $M Y C N$ (Fig. 4E, F). Therefore, MYCN selectively sensitizes NB cells to ferroptosis by increasing the intracellular LIP.

\section{TFRC mediates MYCN induced, GPX4-dependent ferroptosis}

Our data have shown that MYCN upregulates TFRC expression, thereby regulating ferroptosis. Next, we explored whether TFRC mediates GPX4-dependent ferroptosis, in a similar way as $M Y C N$. In $M Y C N$ nonamplified SHEP cells, we found that TFRC overexpression makes cells sensitive to ferroptosis induced by both System $\mathrm{X}_{\mathrm{c}}(-)$ and GPX4 inhibition (Fig. 5A), whereas TFRC knockdown only rescues ferroptosis induced by GPX4 inhibition, but not system $\mathrm{X}_{\mathrm{c}}(-)$ inhibition (Fig. 5B). In SK-N-BE2 cells with amplified $M Y C N$, we also found that knockdown of TFRC rescues the GPX4-dependent but not $\mathrm{X}_{\mathrm{c}}{ }^{-}$-dependent ferroptosis, and the rescue effects are substantially enhanced (Fig. 5C). Moreover, we found that the system $\mathrm{Xc}(-)$ inhibitor SAS upregulates TFRC expression, and TFRC-depleted, MYCN-overexpressed NB cells exhibit high resistance to SAS (Fig. S1E-G). The above results suggest that TFRC indeed mediates GPX4dependent ferroptosis, which can be further reinforced by $M Y C N$ amplification. Consistent with it, the lipid peroxide amount is significantly elevated with ectopic TFRC expression, and decreased upon TFRC knockdown (Fig. 5D, E).

\section{TFRC is a direct downstream target of MYCN}

Given that $M Y C N$ is a well-established transcription factor, we speculated that MYCN drives TFRC expression through direct transcriptional regulation. Indeed, we confirmed that TFRC expression is responsive to either up or down-regulation of $M Y C N$ (Figs. 3C and 6A). Then, we established an inducible $M Y C N-E R$ chimeric protein expression system in SHEP cells, in which nuclear translocation and activity of MYCN can be induced by 4-hydroxytamoxifen (4-HT) treatments. Upon 4-HT stimulation, TFRC expression is elevated at both mRNA and protein levels (Fig. 6B, C), paralleled with lipid peroxide formation in a time-dependent manner (Fig. 6D). To further dissect exactly how $M Y C N$ regulates TFRC expression, we performed ChIPqPCR assays and found that MYCN mainly binds to the $\mathrm{P} 1$ and $\mathrm{P} 2$ regions, which are located $\sim 1.5 \mathrm{~kb}$ away from the transcription starting site (TSS) within the TFRC promoter (Fig. 6E, F). In addition, we cloned the TFRC promoter region from -1973 to $+120 \mathrm{bp}$ surrounding the TSS as shown in Fig. 6E and generated corresponding truncations. We found that $M Y C N$-promoted luciferase signals are strongly attenuated when the region of -1973 to -1623 is eliminated (Fig. 6G), demonstrating that $M Y C N$ regulates TFRC expression by binding to this region. Therefore, we conclude that $M Y C N$ induces GPX4-dependent ferroptosis by upregulating TFRC as a direct downstream target.

\section{Discussion}

The $M Y C$ family member $M Y C N$ plays important role in the development of the central nervous system ${ }^{25,26}$, and its abnormal expression correlates with the poor prognosis of NB patients. Meanwhile, elevated activity of $c-M Y C$, the predominant $M Y C$ isoform, also accelerates the progression of low-grade $\mathrm{NB}^{27}$, indicating that $M Y C$ family members share similar functions during $\mathrm{NB}$ development. Ferroptosis is a novel type of programmed cell death characterized by iron-dependent, unleashed lipid peroxidation, which has been identified in recent years. Cystine deprivation rendered by system $\mathrm{X}_{\mathrm{c}}(-)$ 

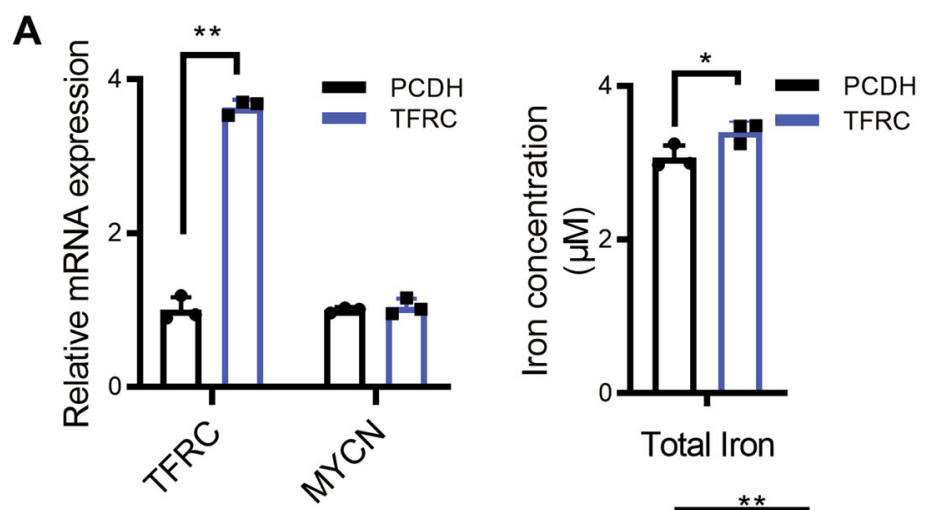

B
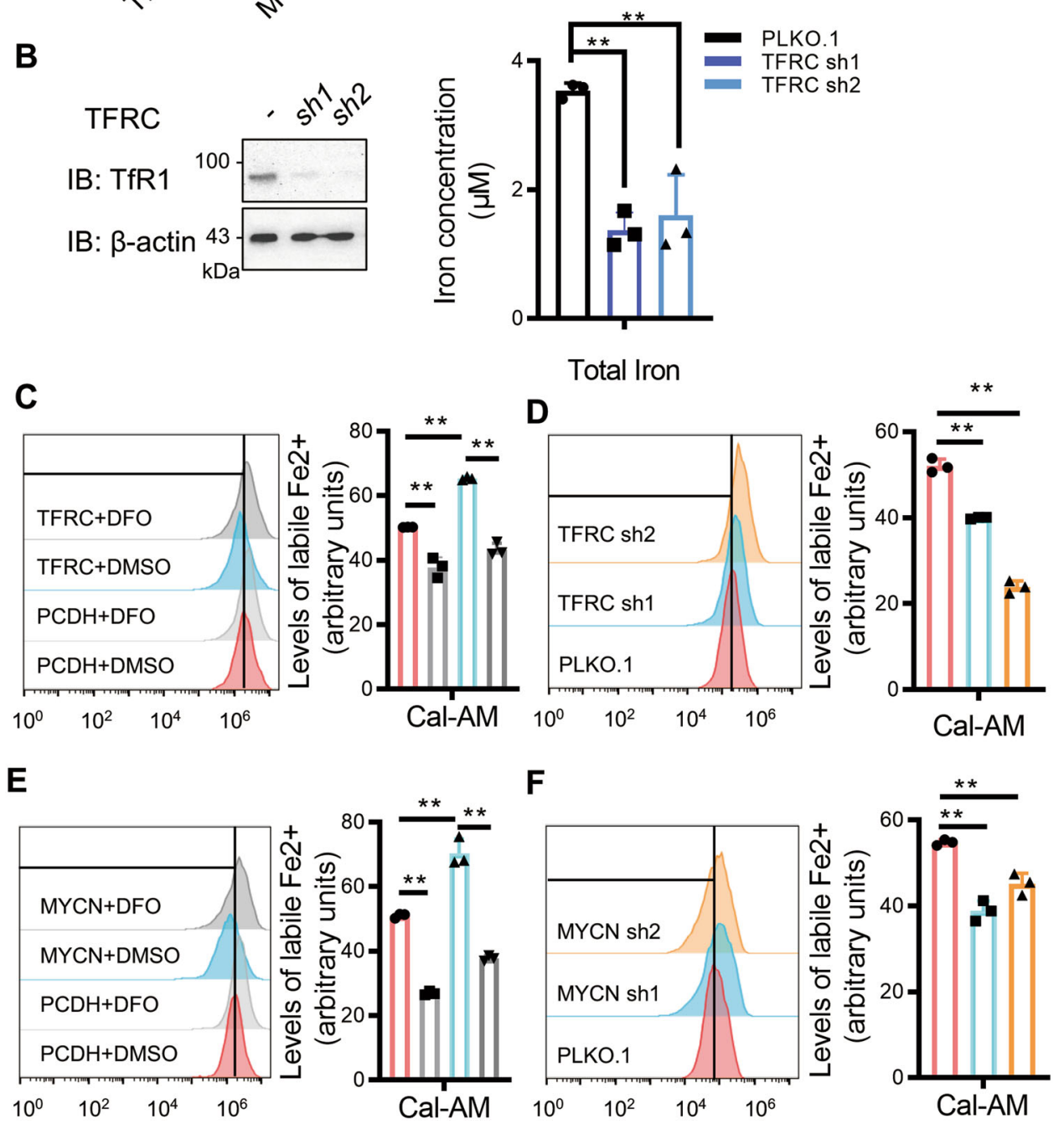

Fig. 4 TFRC photocopies MYCN to increase the labile iron pool. A Iron load and RT-qPCR analysis of SHEP cells with or without TFRC overexpression. B Iron load and western blot analysis of SK-N-BE2 cells with or without TFRC depletion. Cellular labile iron pool (LIP) was detected using the probe calcein acetoxymethyl ester (Cal-AM) in SHEP (C) and SK-N-BE2 (D) cells with or without TFRC. LIP was similarly detected in SHEP (E) and SK-N-BE2 (F) cells with or without MYCN. Data are presented as mean \pm SD of three replicates. Two-tailed unpaired t-tests were performed to calculate $p$ values. ${ }^{*} p<0.05,{ }^{* *} p<0.01$.

blockage, or redox imbalance by GPX4 inhibition are two classic approaches to induce ferroptosis ${ }^{15}$. Our study revealed that $M Y C N$ sensitizes NB cells to ferroptosis, which is largely dependent on GPX4 inhibition rather than cystine deprivation. This is mainly because the abnormal level of MYCN stimulates the expression of 

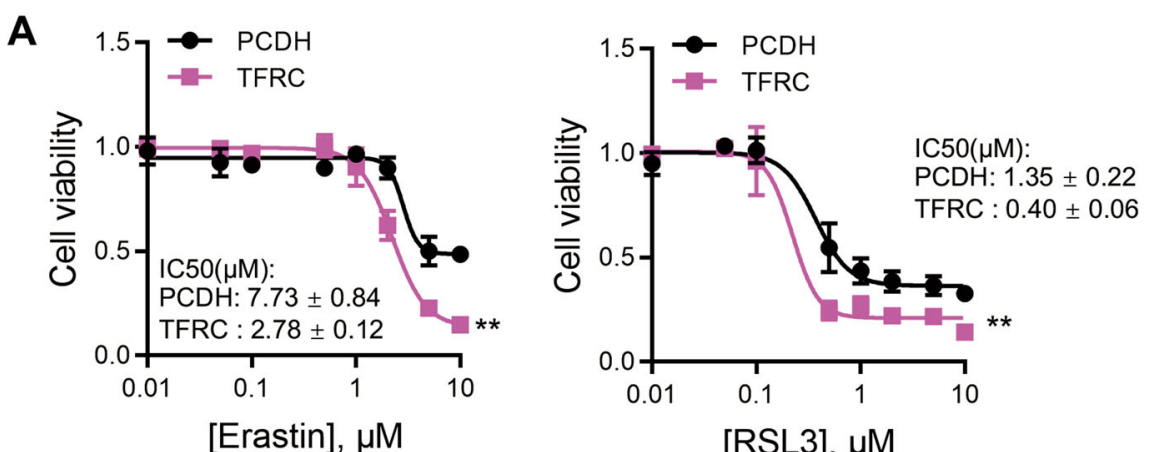

[Erastin], $\mu \mathrm{M}$

B
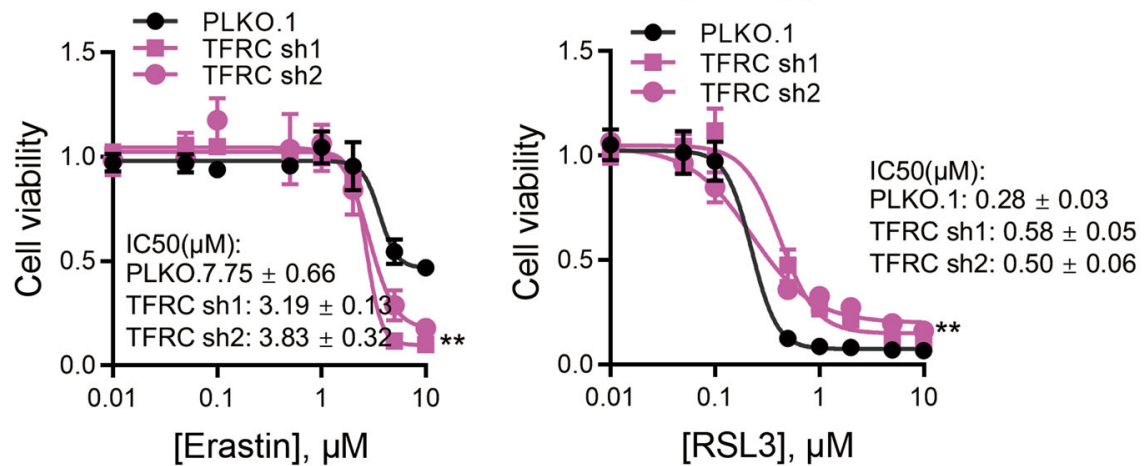

C

[Erastin], $\mu \mathrm{M}$
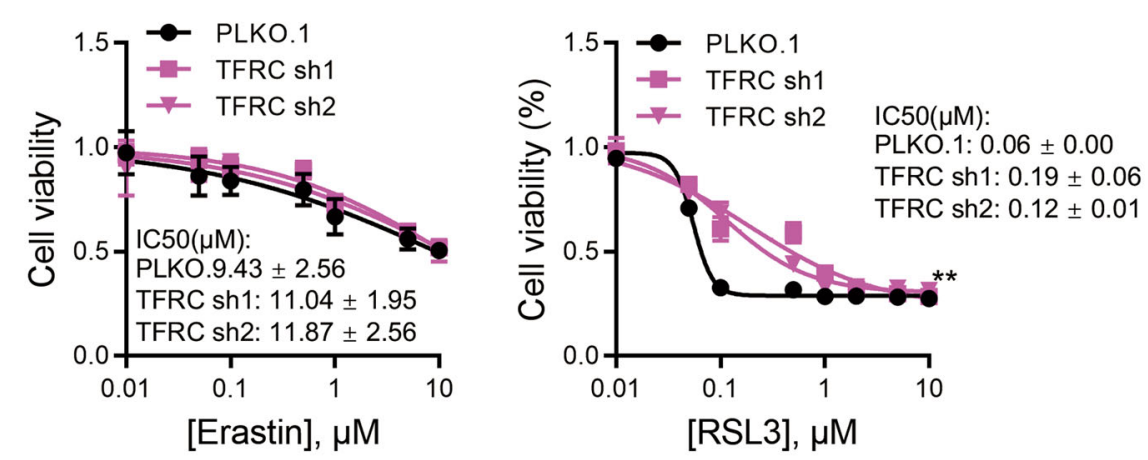

D

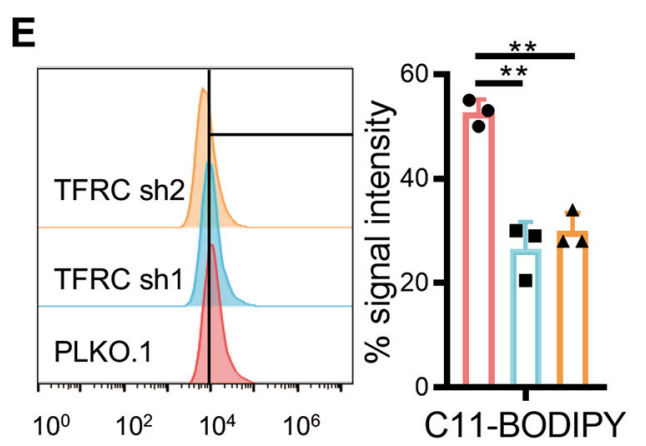

Fig. 5 TFRC mediates MYCN-induced, GPX4-dependent ferroptosis. A Cell viability of SHEP cells with or without TFRC overexpression incubated with Erastin (left) or RSL3 (right). Cell viabilities of SHEP (B) or SK-N-BE2 (C) cells with or without TFRC depletion incubated with Erastin (left) or RSL3 (right). Amounts of cellular lipid peroxides measured by C11-BODIPY in SHEP (D) or SK-N-BE2 (E) cells with or without TFRC. Data are presented as mean \pm SD of three replicates. Two-tailed unpaired $t$-tests were performed to calculate $p$ values. ${ }^{* *} p<0.01$.

TFRC, increases the intracellular levels of iron load and LIP, ultimately leads to enhanced lipid peroxidation and a tendency towards ferroptosis.
One important function of $M Y C N$ in NB is upregulating glutamine transporters, such as ASCT2, thereby promoting glutamine addiction ${ }^{28,29}$, a hallmark of tumor 


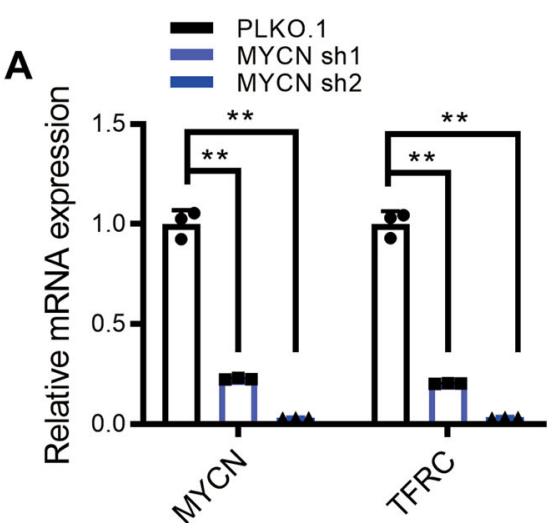

B

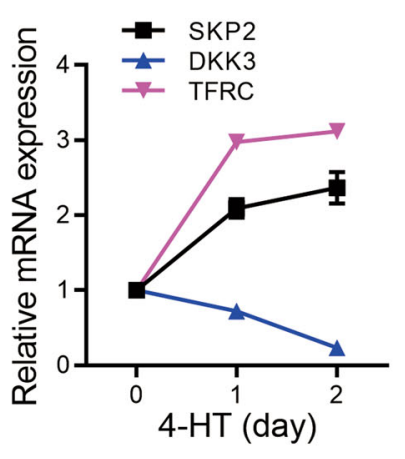

C

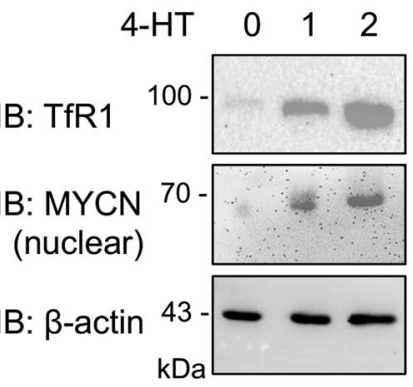

D
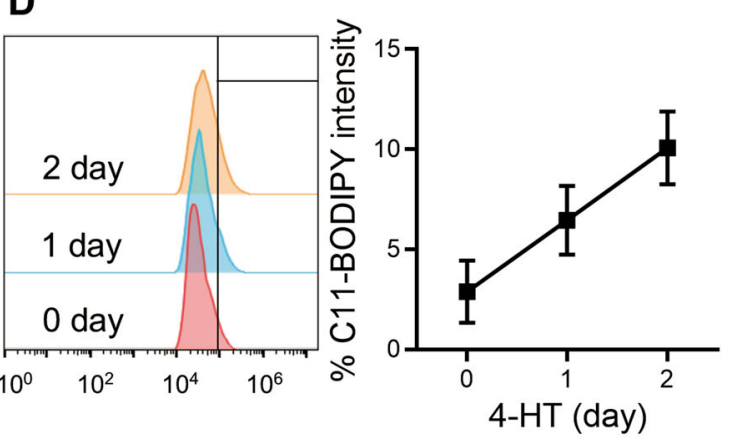

E

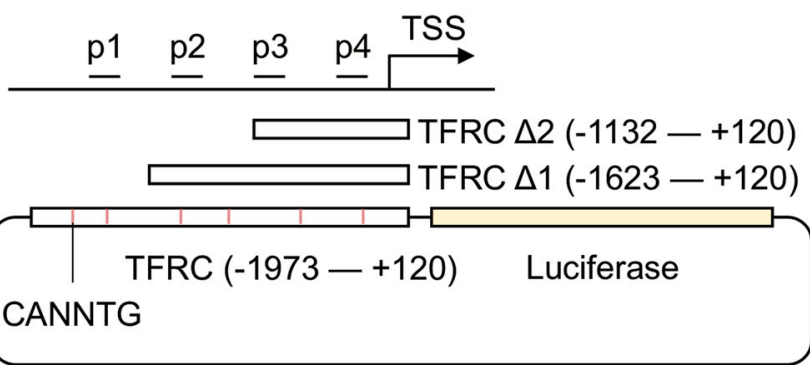

$\mathbf{F}$

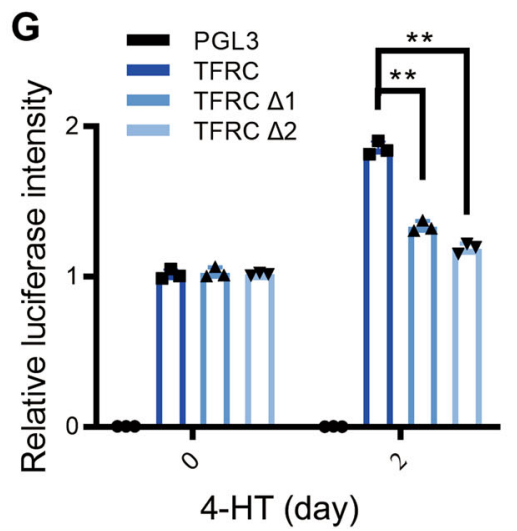

Fig. 6 TFRC is a direct downstream target of MYCN. A RT-qPCR analysis of SK-N-BE2 cells with or without MYCN depletion. MYCN activation induced by 4-hydroxytamoxifen (4-HT) treatment promotes TFRC expression at both mRNA B and protein C levels. SKP2, DKK3 were used as positive and negative controls, respectively. D Amounts of cellular lipid peroxides measured by C11-BODIPY upon MYCN activation. E Primers designed for ChIP-qPCR analysis, and sequence variants for luciferase assays based on the TFRC promoter region. F ChIP-qPCR analysis of different binding sites of MYCN on the TFRC promoter. MDM2 is a positive control for MYCN binding. $\mathbf{G}$ Dual-luciferase assays based on (E). Signal values were normalized to no 4-HT treatment (day 0). Data are presented as mean \pm SD of three replicates. Two-tailed unpaired $t$-tests were performed to calculate $p$ values. ${ }^{* *} p<$ 0.01 . 
metabolic reprogramming. In NB cells, the glutamic acid level is decreased upon $M Y C N$ amplification (Fig. 2E), confirming that $M Y C N$ promotes glutamine consumption. It was reported that glutaminolysis and iron are both essential for Erastin-induced ferroptosis in an ischemia/ reperfusion model ${ }^{30}$, implying that $M Y C N$ might induce ferroptosis through system $\mathrm{X}_{c}(-)$ in that context. However, our results reveal that $M Y C N$ induces ferroptosis in NB cells independent of system $X_{c}(-)$, suggesting that the molecular mechanism underlying $M Y C N$-induced ferroptosis is highly context-dependent.

Notably, Floros et al. recently reported that $M Y C N$ amplified NB cells are more sensitive to the system $\mathrm{X}_{\mathrm{c}}(-)$ inhibitor sulfasalazine (SAS) ${ }^{31}$. Indeed, $M Y C N$-amplified NB cells are slightly sensitive to SAS as reflected by their smaller IC50 values compared to non-amplified cells, although no statistical significance was achieved in our hands (Fig. S1C). This trend is seemingly contradictory to what we observed with Erastin. However, SAS equally inhibits other targets such as $\mathrm{NFKB}$ and reduced folate carrier $^{32,33}$ in addition to System $\mathrm{X}_{\mathrm{c}}(-)$, which may help explain this discrepancy. Interestingly, Floros et al. noticed that SAS treatment of $M Y C N$-amplified NB in vivo upregulate TFRC expression through an uncharacterized mechanism, which can be reproduced by us in vitro (Fig. S1E). Since we demonstrated that enhanced TFRC expression is responsible for increased cellular sensitivity to ferroptosis, we reasoned that SAS may exert its effect through a similar mechanism. Indeed, TFRCdepleted, MYCN-overexpressed NB cells exhibit high resistance to SAS (Fig. S1F, G), demonstrating that SAS sensitizes $M Y C N$-amplified NB cells to ferroptosis at least partially through TFRC upregulation. This argument can be further supported by the study from Floros et al. For instance, SAS is capable of inducing $~ 70 \%$ cell death in $M Y C N$-amplified NB cells. However, knockdown of two System $\mathrm{X}_{\mathrm{c}}(-)$ components SLC7A11 and SLC3A2 induce only $\sim 25 \%$ cell death in the same cell line ${ }^{31}$, confirming that SAS harbors considerable off-target effects. In conclusion, the complex effects of SAS treatments leading to ferroptosis cannot be solely linked to the sensitivity of NB cells to System $\mathrm{X}_{\mathrm{c}}(-)$ inhibition.

There are multiple major differences between our studies and the work by Floros et al. First, they were focused on targeting the redox balance of $M Y C N$-amplified NB cells to induce ferroptosis, using different small molecule inhibitors such as buthionine-(S,R)-sulfoximine (BSO), sulfasalazine (SAS), and auranofin. All these inhibitors function to antagonize the ROS-scavenging activities in $M Y C N$-amplified NB cells. In contrast, our work highlights TFRC as the major downstream target of $M Y C N$ to confer cell sensitivities to ferroptosis inhibitors. We particularly demonstrated that TFRC depletion greatly suppresses $M Y C N$-mediated ferroptosis, as companied by the reduction of the intracellular iron pool. Moreover, TFRC overexpression phenocopies $M Y C N$ amplification to increase the intracellular iron pool and sensitize these tumor cells to ferroptosis. Given that ferroptosis requires both elevated iron levels and dampened ROS-neutralizing activities, these two studies compensate with each other in understanding the vulnerability of $M Y C N$-amplified NB cells towards ferroptosis. Second, work by Floros et al. suggested that MYCN binds to the TFRC promoter and upregulates $T F R C$ expression by analyzing relevant data in a public ChIP-seq database. Conversely, we performed ChIPPCR analyses to identify the $M Y C N$-binding region within the TFRC promoter, and further confirmed this result by luciferase reporter assays. Third, they found that $M Y C N$ amplified NB cells are sensitive to the System $X_{c}(-)$ inhibitor SAS, whereas our study suggested that these cells are resistant to the System $X_{c}(-)$ inhibitor Erastin.

Iron is an essential element for cell growth and survival, the deregulation of which determines cell fate. In the process of ferroptosis, iron acts as a catalyst for lipid peroxidation through Fenton reactions ${ }^{23}$. TfR 1 encoded by TFRC is the main iron transporter in cells, and it has been reported that SLC39A14 is an additional iron transporter ${ }^{34}$, although our experiment shows that $M Y C N$ fails to regulate SLC39A14 expression (Fig. 3C). In addition, several classical $M Y C N$ binding motifs (CANNTG) are present in the TFRC promoter, and our ChIP-qPCR analysis reveals that $M Y C N$ precisely binds to a motif-containing region distal to TSS. The specific recognition and regulation of TFRC by MYCN is consistent with previous findings ${ }^{35}$. Recently, the Stockwell group reported that TfR1 clusters on the cell membrane can be used as a detection marker for ferroptosis ${ }^{36}$. They observed that TfR 1 expression is increased during ferroptosis induction, but the mechanism is unknown. Our study supports the MYC signaling as an important candidate. Moreover, it has been reported that TfR1 depletion confers cell resistance to Erastin-induced ferroptosis under abnormal RAS activities ${ }^{37}$. However, we found that TFRC knockdown has no significant impact on Erastin-induced ferroptosis in $M Y C N$-amplified cells. These results suggest that Erastin-induced ferroptosis harbors a regulatory mechanism independent of TFRC, which is likely decided by different oncogenic signals. Although MYC is one of the first identified oncogenes ${ }^{38,39}$, it can function to induce p53dependent cell death, similar to the case that oncogenic RAS can initiate cell senescence ${ }^{40,41}$. Whether and how different oncogenes cooperate to determine cancer cell fate is pivotal for developing new cancer therapeutics.

\section{Materials and methods Cell culture}

Human NB cell lines with MYCN amplification (SK-NBE2, BE(2)-C, NLF, SK-N-DZ, Kelly) and without MYCN amplification (SHEP, SK-N-AS, SY5Y) were grown in 
RPMI 1640 medium supplemented with 10\% FBS and 1\% penicillin-streptomycin, under $5 \% \mathrm{CO}_{2}$ at $37^{\circ} \mathrm{C}$. Cell authentication was confirmed by the short tandem repeat analysis, and mycoplasma tests were weekly performed. Cell stocks were created within five passages, and experiments were completed within ten passages.

\section{Antibodies and reagents}

Antibodies and reagents were obtained from commercial sources. Specifically, anti-transferrin receptor, rabbit antibody (ab84036) was from Abcam; N-Myc (D1V2A) rabbit mAb (84406), beta-actin (8H10D10) mouse mAb, HRP-linked mouse IgG (7076 S), and rabbit IgG (7074S) were from Cell Signaling Technology (Massachusetts, USA). Erastin (HY-15763), (1 S,3 R)-RSL3 (HY-100218A), deferoxamine mesylate (DFO), Z-VAD(OMe)-FMK(HY16658), Necrostatin-1 (HY-15760) were from MedChemExpress (New Jersey, USA); (Z)-4-hydroxytamoxifen (T4420) and sulfasalazine (SAS, T0907) from Target Molecule Corp. (Massachusetts, USA); BODIPY ${ }^{\mathrm{TM}}$ 581/591 C11 (D3861), Cal-AM (C3099), Ferrozine (160601), and Neocuproine (N1501) from Invitrogen, Thermofisher (California, USA). Polyethylenimine (PEI) Linear, MW 25000 (24769-2) was from Polysciences Inc (Pennsylvania, USA). CCK-8 reagent is from Beyotime Inst Biotech (C0040, Shanghai, China).

\section{Constructs}

$M Y C N$ and TFRC expression plasmids were constructed by cloning the open reading frame of the corresponding cDNA into the multiple cloning sites of the $\mathrm{PCDH}$ vector. shRNA sequences were cloned into PLKO.1 vector according to Addgene's protocol. For the luciferase assay, promoter sequences were cloned into pGL3 Luciferase Reporter Vectors (E1741, Promega, Wisconsin, USA).

\section{RNA-seq analysis}

Total RNA was extracted from each sample using the standard TRIzol protocol. The Agilent 2100 Bioanalyzer was employed to verify RNA quality. cDNA libraries were generated and processed for sequencing following the Illumina TrueSeq version 2 library preparation kit's protocol. The cDNA libraries were then sequenced by Illumina HiSeq 2500 with paired-end $2 \times 75$ bp reads using the HiSeq Control Software (version 2.0.10). The quality of raw reads was evaluated by FastQC, and reads alignment was performed using STAR (version 2.3.0). We used SAMTools to sort the aligned reads, and performed genelevel read quantifications with HTSeq (version 0.10.0).

\section{Bioinformatic analyses}

Gene expression correlation and survival analyses for NB patients (retrieved from GSE45547 ${ }^{42}$ and
GSE49710 ${ }^{43}$ ) were conducted using the R2 Platform (https://hgserver1.amc.nl/cgi-bin/r2/main.cgi), and the resulting data and $\mathrm{p}$ values were calculated according to online instructions. Gene Ontology (GO) analysis of MYCN-dependent genes ${ }^{17}$ was performed using the Molecular Signatures Database v7.2 online tool (http:// www.gsea-msigdb.org/gsea/msigdb/annotate.jsp). For GSEA analysis, the expression data of six NB cells by RNA-seq were used, and cell lines were divided into two groups based on the conventional MYCN status (MYCN amplified: SK-N-BE2, BE(2)-C, NLF, SK-N-DZ v.s. $M Y C N$ non-amplified: SHEP, SK-N-AS). Lists and expression data of ferroptosis-related and $M Y C N$-dependent survival genes are provided in Supplementary Tables 1 and 2.

\section{Viral production and transduction}

HEK-293T cells were seeded on a 10-cm culture dish with $50 \%$ confluence. After attachment, cells were cotransfected with the lentiviral expression plasmid $(10 \mu \mathrm{g})$, viral packaging plasmid (psPAX2, Addgene 12260, $5 \mu \mathrm{g}$ ), and envelope plasmid (pMD2.G, Addgene 12259, $2 \mu \mathrm{g}$ ) using PEI following the manufacturer's protocols. Viral supernatants were collected and filtered after transfection. For viral transduction, target cells were seeded and incubated with a medium supplemented with viral particles and $8 \mathrm{mg} / \mathrm{mL}$ polybrene for $6-8 \mathrm{~h}$. Virus-infected cells were selected with corresponding antibiotics.

\section{Cell viability assay}

Cell viability was detected with CCK- 8 according to the manufacturer's protocol. Briefly, $5 \times 10^{3}$ cells were seeded in 96-well plates per well and treated with different compounds. Twenty-four hours later, cells are incubated with CCK-8 for $2 \mathrm{~h}$ at $37^{\circ} \mathrm{C}$. Viability values were obtained by a plate reader at $450 \mathrm{~nm}$.

\section{Total iron analysis}

Total iron concentrations of cells were measured by a colorimetric ferrozine-based assay as previously reported $^{44}$. Briefly, $5 \times 10^{5}$ cells were seeded in $6 \mathrm{~cm}$ dishes for $24 \mathrm{~h}$, collected with $50 \mathrm{mM} \mathrm{NaOH}$, and incubate at room temperature (RT) for $2 \mathrm{~h}$. Iron ion was extracted with iron-releasing buffer $\left(1.4 \mathrm{M} \mathrm{HCl}\right.$ with $\left.4.5 \% \mathrm{KMnO}_{4}, 1: 1\right)$ at $60^{\circ} \mathrm{C}$ for $2 \mathrm{~h}$, followed by incubation with $30 \mu \mathrm{L}$ detecting buffer (6.5 mM Ferrozine, $6.5 \mathrm{mM}$ Neocuproine, $2.5 \mathrm{M}$ ammonium acetate, $1 \mathrm{M}$ ascorbic acid) at RT for $30 \mathrm{~min}$. Absorbance was measured with a 96-well plates reader at $550 \mathrm{~nm}$. The iron contents were calculated based on $\mathrm{FeCl}_{3}$ standard curves and normalized to protein concentration.

\section{Labile iron pool (LIP) analysis}

Calcein acetoxymethyl ester (Cal-AM) is a low-toxic and non-fluorescence dye that can easily pass through the 
cell membrane. Cal-AM emits fluorescence after being digested by esterase in living cells and quenched in the presence of $\mathrm{Fe}^{2+}$, which can be used as a LIP probe as previously described ${ }^{24}$. In this study, $2 \times 10^{5}$ cells were seeded on six-well plates for $24 \mathrm{~h}$, after washing with PBS three times, and incubated with $20 \mathrm{nM}$ Cal-AM in HBSS at $37^{\circ} \mathrm{C}$ for $30 \mathrm{~min}$. Then, Cells were trypsinized, suspended, and analyzed using a BD Accuri C6 flow cytometer with a $488 \mathrm{~nm}$ laser on an FL1 detector.

\section{Measurement of lipid peroxides}

Cells were incubated with $200 \mathrm{nM}$ C11-BODIPY in HBSS at $37^{\circ} \mathrm{C}$ for $30 \mathrm{~min}$, and analyzed using a BD Accuri C6 flow cytometer with a $488 \mathrm{~nm}$ laser on an FL1 detector.

\section{ChIP-qPCR}

Assays were performed by following a modified Abcam's Chromatin immunoprecipitation protocol. Briefly, cells overexpressing biotinylated-tagged proteins (avi tag) were fixed in 1\% formaldehyde at RT for $10 \mathrm{~min}$ and stopped by the addition of $0.125 \mathrm{M}$ glycine. Cells were then harvested in ChIP Lysis Buffer (50 mM HEPES-KOH $\mathrm{pH}=7.5,140 \mathrm{mM} \mathrm{NaCl}, 1 \mathrm{mM}$ EDTA, $1 \%$ Triton X-100, $0.1 \%$ sodium deoxycholate, $0.1 \%$ SDS, $1 \mathrm{X}$ protease inhibitor cocktail). Lysates were sonicated and DNA sheared to an average length of $200-500 \mathrm{bp}$. Genomic DNA (Input) was prepared by treating aliquots of chromatin with RNase A, Proteinase $\mathrm{K}$, and $65^{\circ} \mathrm{C}$ heat for decrosslinking, followed by ethanol precipitation. The resuspended pellet was incubated with magnetic streptavidin beads (Z5482, Promega, Wisconsin, USA) for $4 \mathrm{~h}$ rocking at $4{ }^{\circ} \mathrm{C}$, washed four times, and eluted from beads with SDS elution buffer (1\% SDS, $10 \mathrm{mM}$ EDTA, $50 \mathrm{mM}$ Tris- $\mathrm{HCl} \mathrm{pH}=8$ ). Crosslinks were reversed by high salt incubation for $4 \mathrm{~h}$ at $65^{\circ} \mathrm{C}$. ChIP DNA was purified by phenol-chloroform extraction, followed by quantitative real-time PCR analysis. Primers used for ChIP-qPCR:

TFRC p1: fw: TGAGGTCAGGAATTCGAGACAAG; rev: GAGGAGTGACGGGATTTTATC;

TFRC p2: fw: GCAGCAATGCTCCTGCTCA; rev: GC GACAGAGCAAGATTCCATC;

TFRC p3: fw: GGGAAGAGTAAAAGCCCAAGG; rev: GAACAGCCCTTTAAGAAGCAAATC;

TFRC p4: fw: GGCTGCAAAATACATCTTCACAAG; rev: GCCTTGAAATGTACGTGCAGG;

MDM2 promoter fw: AGCCTTTGTGCGGTTCGTG; rev: CCCCCGTGACCTTTACCCTG.

\section{Quantitative real-time PCR analysis}

Total RNA was extracted from cells using the RNA Midiprep kit (AP-MN-MS- RNA-250, Axygen, California, USA) according to the manufacturer's protocol. Then, cDNAs were generated using a Reverse Transcription kit
(RR036A, Takara, Beijing, China). Quantitative real-time PCR was performed using the TB Green Premix Ex Taq qPCR kit (AQ101, Transgen Biotech, Beijing, China). Primers used for RT-qPCR:

18S: fw: CTACCACATCCAAGGAAGGCAG; rev: TTT TTCGTCACTACCTCCCCG;

MYCN:fw: CCACAAGGCCCTCAGTACC; rev: TCTT CCTCTTCATCATCTTCATCA;

GPX4:fw: TGGGAAATGCCATCAAGTG; rev: GGGG

CAGGTCCTTCTCTATC;

SLC11A2: fw: CTGCACCATGAGGAAGAAGC; rev: TGGATACCTGAGTGGCTGAGT;

SLC40A1: fw: CCCCAGCTCTAGCTGTGAAA; rev: CAGGGGTTTTGGCTCAGTAT;

IREB1: fw: CCTCAGCCCCTGTCAAAA; rev: GATT ACTGATGGCCACGTGTT;

IREB2:fw: AGAAATATGGTTCAGGAAACTCCA; rev: GCCAAAACAGCTTTCACACC;

SLC39A14: fw: AAGGCCCTACTCAACCACCT; rev: CGACTGCTCGCTGAAATTGTG

TFRC: fw: ACCTGTCCAGACAATCTCCAG; rev: TG TTTTCCAGTCAGAGGGACA;

DKK3: fw: GAAGGAGCCACGAGTGCAT; rev: CCTC ATGCTGTCAAGCCAGA;

SKP2: fw: GACGCTATGCACAGGAAGCA; rev: CCTT TAGCAGCTCAGGGAGG.

\section{Luciferase reporter assay}

Dual-Luciferase Reporter Assay System (E1910, Promega, Wisconsin, USA) was used. Briefly, pGL3 vectors carrying TFRC promoter sequences were transfected into SHEP cells. Cells were then transferred to six-well plates with $2 \times 10^{5}$ per well. MYCN activity was induced with $40 \mathrm{nM} 4$-HT treatments. Luciferase signals were measured $48 \mathrm{~h}$ later. Firefly luciferase signals were divided by Renilla luciferase signals, and rescaled to set the control signals equal to 1 .

Primers for cloning the TFRC promoter: fw: CCTGC AAATACCAGCATTGTTTG; TFRC $\Delta$ 1: fw GAAGGAA CACCACAGGGGAGCA; TFRC $\triangle 2$ fw: GGCGGTGAGA ATCCCAAGTACT; The reverse primer for all above: GACACGAGGGTCGGTGTAGTTC.

\section{Western blot}

Briefly, cells were lysed with RIPA buffer $(20 \mathrm{mM}$ Tris$\mathrm{HCl} \mathrm{pH} 7.4,150 \mathrm{mM} \mathrm{NaCl}, 1 \mathrm{mM}$ EDTA, $0.5 \%$ sodium deoxycholate, $0.1 \%$ SDS, $1 \%$ Triton-X) supplemented with the protease inhibitor cocktail. Lysates were resolved on SDS-PAGE followed by immunoblotting. Primary antibodies were used following instructions in the antibody dilution buffer (1\% BSA, $0.05 \%$ sodium azide in $0.05 \mathrm{M}$ TBS, $\mathrm{pH}=7.6$ ). ChemiDoc ${ }^{\mathrm{TM}}$ Touch Imaging System (Bio-Rad, California, USA) was used to develop the blot on the $0.2 \mu \mathrm{m}$ diameter PVDF membrane. 


\section{Metabolic analysis}

For GC-MS assays, cells were washed with pre-cold physiological saline buffer and harvested in HPLC grade methanol containing internal standards (Ribitol). After sonication and $20,000 \mathrm{~g}$ centrifugation at $4{ }^{\circ} \mathrm{C}$, the supernatants were transferred to new $1.5 \mathrm{~mL}$ tubes. The supernatants were then transferred into the rotary evaporator and evaporate completely. After treatment with derivatization reagent, samples were analyzed with GCMS according to the manufacturer protocol. For LC-MS assays, cells were washed with pre-cold physiological saline buffer and harvested in HPLC grade methanol. Then, lipids were extracted with chloroform after 12,000 g centrifugation at $4{ }^{\circ} \mathrm{C}$. The lower phase was evaporated with nitrogen and esterified in 5\% sulfuric acid in methanol. The extracts were analyzed by capillary electrophoresis time-of-flight mass spectrometry. Peak areas were normalized to standards and cell numbers.

\section{Statistical analysis}

Data were presented as mean \pm SD. Statistical significance was analyzed using a two-tailed unpaired $t$-test and assessed by $p$ values as reported in figure legends. Kaplan-Meier srvival analyses employed the log-rank test.

\section{Acknowledgements}

We thank the Metabolic Innovation Centre at Sun Yat-sen University for assistance in metabolic and fatty acid assays.

\section{Author details}

${ }^{1}$ Clinical Biological Resource Bank, Guangzhou Institute of Pediatrics, Guangzhou Women and Children's Hospital, Zhongshan School of Medicine, Sun Yat-sen University, Guangzhou, China. ${ }^{2}$ State Key Laboratory of Oncology in South China, Collaborative Innovation Center for Cancer Medicine, Sun Yatsen University Cancer Center, Guangzhou, China. ${ }^{3}$ Department of Biochemistry, Zhongshan School of Medicine, Sun Yat-sen University, Guangzhou, China. ${ }^{4}$ Key Laboratory of Functional Protein Research of Guangdong Higher Education Institutes, Department of Biotechnology, College of Life Science and Technology, Jinan University, Guangzhou, China. ${ }^{5}$ State Key Laboratory of Translational Medicine and Innovative Drug Development, Simcere Diagnostics Co., Ltd., Nanjing, China. ' Department of Neurosurgery, Jiangmen Central Hospital, Affiliated Jiangmen Hospital, Sun Yat-sen University, Jiangmen, China. ${ }^{7}$ Center for Precision Medicine, Sun Yat-sen University, Guangzhou, China. ${ }^{8}$ RNA Biomedical Institute, Sun Yat-sen Memorial Hospital, Sun Yat-sen University, Guangzhou, China

\section{Author contributions}

Conceived the study and analyzed the data: Z.L., J-X.B., C.P., X.G. and B.L. Designed and performed the experiments: Y.L., Q.Y., Y.S., Y.J., G.L., X.Y., L.X., J.D. and Y.W., Wrote the manuscript: C.P., X.G., and B.L.

\section{Funding}

This study was supported by the National Key Research and Development Program of China (2016YFA0502600 to B.L.); the National Natural Science Foundation of China (82073074 to B.L., 42076095 to Y.S., and 82003194 to C.P.); Guangdong Natural Science Fund, China (2021A1515011207 to X.G.); the National Program for Support of Top-Notch Young Professionals (to J-X.B.); Chang Jiang Scholars Program (to J-X.B.); Special Support Program of Guangdong (to J-X.B.); Sun Yat-sen University Young Teacher Key Cultivate Project (17ykjc24); Guangdong Innovative and Entrepreneurial Research Team Program (2016ZT06S638); the postdoctoral research initiation fund by Guangzhou Institute of Pediatrics, Guangzhou Women and Children's Medical Center.

\section{Data availability}

Our raw RNA seq deposited in NCBI SRA database, and the accession ID is PRJNA720775

\section{Ethics statement}

We do not process samples of human participants in this study. Analyzed gene expressions of human NB tissues were obtained from a public database, which was approved by the Ethics Committee of the Medical Faculty of University of Cologne or local universities of collaborators according to related reference. The study was performed in accordance with the Declaration of Helsinki.

\section{Conflict of interest}

The authors declare no competing interests.

\section{Publisher's note}

Springer Nature remains neutral with regard to jurisdictional claims in published maps and institutional affiliations.

Supplementary information The online version contains supplementary material available at https://doi.org/10.1038/s41419-021-03790-w.

Received: 6 February 2021 Revised: 29 April 2021 Accepted: 29 April 2021 Published online: 19 May 2021

\section{References}

1. Maris, J. M., Hogarty, M. D., Bagatell, R. \& Cohn, S. L. Neuroblastoma. Lancet 369, 2106-2120 (2007).

2. Matthay, K. K. et al. Neuroblastoma. Nat. Rev. Dis. Prim. 2, 16078 (2016).

3. Diskin, S. J. et al. Common variation at $6 q 16$ within HACE1 and LIN28B influences susceptibility to neuroblastoma. Nat. Genet. 44, 1126-1130 (2012).

4. Rickman, D. S., Schulte, J. H. \& Eilers, M. The expanding world of N-MYC-driven tumors. Cancer Discov. 8, 150-163 (2018).

5. Ruiz-Perez M. V., Henley A. B. Arsenian-Henriksson M. The MYCN protein in health and disease. Genes 8, 113 (2017).

6. Huang, M. \& Weiss, W. A. Neuroblastoma and MYCN. Cold Spring Harb. Perspect. Med. 3, a014415 (2013).

7. Yin, Y. et al. N-Myc promotes therapeutic resistance development of neuroendocrine prostate cancer by differentially regulating miR-421/ATM pathway. Mol. Cancer 18, 11 (2019).

8. Olsen, R. R. et al. MYCN induces neuroblastoma in primary neural crest cells. Oncogene 36, 5075-5082 (2017).

9. McMahon, S. B. MYC and the control of apoptosis. Cold Spring Harb. Perspect. Med. 4, a014407 (2014).

10. King, D. et al. MYCN expression induces replication stress and sensitivity to PARP inhibition in neuroblastoma. Oncotarget 11, 2141-2159 (2020).

11. Feng, H. \& Stockwell, B. R. Unsolved mysteries: how does lipid peroxidation cause ferroptosis? PLoS Biol. 16, e2006203 (2018).

12. Miess, $\mathrm{H}$. et al. The glutathione redox system is essential to prevent ferroptosis caused by impaired lipid metabolism in clear cell renal cell carcinoma. Oncogene 37, 5435-5450 (2018).

13. Chekhun, V. F. et al. Iron metabolism disturbances in the MCF-7 human breas cancer cells with acquired resistance to doxorubicin and cisplatin. Int. J. Oncol. 43, 1481-1486 (2013)

14. Alvarez, S. W. et al. NFS1 undergoes positive selection in lung tumours and protects cells from ferroptosis. Nature 551, 639-643 (2017).

15. Stockwell, B. R. \& Jiang, X. The chemistry and biology of ferroptosis. Cell Chem Biol. 27, 365-375 (2020).

16. Tjaden, B. et al. N-Myc-induced metabolic rewiring creates novel therapeutic vulnerabilities in neuroblastoma. Sci. Rep. 10, 7157 (2020).

17. Durbin, A. D. et al. Selective gene dependencies in MYCN-amplified neuroblastoma include the core transcriptional regulatory circuitry. Nat. Genet. 50, 1240-1246 (2018).

18. Liang, J. Y. et al. A novel ferroptosis-related gene signature for overall survival prediction in patients with hepatocellular carcinoma. Int. J. Biol. Sci. 16, 2430-2441 (2020).

19. Hadian, K. \& Stockwell, B. R. SnapShot: ferroptosis. Cell 181, 1188-1188.e1 (2020). 
20. Shukla, $\mathrm{K}$. et al. Inhibition of $\mathrm{xc}^{-}$transporter-mediated cystine uptake by sulfasalazine analogs. Bioorg. Med. Chem. Lett. 21, 6184-6187 (2011).

21. Xia, Y. et al. Metabolic reprogramming by MYCN confers dependence on the serine-glycine-one-carbon biosynthetic pathway. Cancer Res. 79, 3837-3850 (2019).

22. Dixon, S. J. \& Stockwell, B. R. The hallmarks of ferroptosis. Annu. Rev. Cancer Biol. 3, 35-54 (2019).

23. Hassannia, B., Vandenabeele, P. \& Vanden Berghe, T. Targeting ferroptosis to iron out cancer. Cancer Cell 35, 830-849 (2019).

24. Liu, X. et al. Cystine transporter regulation of pentose phosphate pathway dependency and disulfide stress exposes a targetable metabolic vulnerability in cancer. Nat. Cell Biol. 22, 476-486 (2020).

25. Knoepfler, P. S., Cheng, P. F. \& Eisenman, R. N. N-myc is essential during neurogenesis for the rapid expansion of progenitor cell populations and the inhibition of neuronal differentiation. Genes. Dev. 16 2699-2712 (2002).

26. Sawai, S. et al. Defects of embryonic organogenesis resulting from targeted disruption of the N-myc gene in the mouse. Development 117, 1445-1455 (1993).

27. Fredlund, E., Ringner, M., Maris, J. M. \& Pahlman, S. High Myc pathway activity and low stage of neuronal differentiation associate with poor outcome in neuroblastoma. Proc. Natl Acad. Sci. USA 105, 14094-14099 (2008).

28. Ren, P. et al. ATF4 and N-Myc coordinate glutamine metabolism in MYCNamplified neuroblastoma cells through ASCT2 activation. J. Pathol. 235, 90-100 (2015).

29. Qing, G. et al. ATF4 regulates MYC-mediated neuroblastoma cell death upon glutamine deprivation. Cancer Cell 22, 631-644 (2012).

30. Gao, M., Monian, P., Quadri, N., Ramasamy, R. \& Jiang, X. Glutaminolysis and transferrin regulate ferroptosis. Mol. Cell 59, 298-308 (2015).

31. Floros K. V., et al. MYCN-amplified neuroblastoma is addicted to iron and vulnerable to inhibition of the system $X_{c}$-/glutathione axis. Cancer Res. https://doi.org/10.1158/0008-5472.CAN-20-1641 (2021).
32. Weber, C. K., Liptay, S., Wirth, T., Adler, G. \& Schmid, R. M. Suppression of NFkappaB activity by sulfasalazine is mediated by direct inhibition of IkappaB kinases alpha and beta. Gastroenterology 119, 1209-1218 (2000).

33. Jansen, $G$. et al. Sulfasalazine is a potent inhibitor of the reduced folate carrier: implications for combination therapies with methotrexate in rheumatoid arthritis. Arthritis Rheum. 50, 2130-2139 (2004).

34. Jenkitkasemwong, S. et al. SLC39A14 is required for the development of hepatocellular iron overload in murine models of hereditary hemochromatosis. Cell Metab. 22, 138-150 (2015).

35. Wang, T. et al. MYCN drives glutaminolysis in neuroblastoma and confers sensitivity to an ROS augmenting agent. Cell Death Dis. 9, 220 (2018).

36. Feng, H. et al. Transferrin receptor is a specific ferroptosis marker. Cell Rep. $\mathbf{3 0}$ 3411-3423.e7 (2020)

37. Yang, W. S. \& Stockwell, B. R. Synthetic lethal screening identifies compounds activating iron-dependent, nonapoptotic cell death in oncogenic-RASharboring cancer cells. Chem. Biol. 15, 234-245 (2008).

38. Adams, J. M. et al. The c-myc oncogene driven by immunoglobulin enhancers induces lymphoid malignancy in transgenic mice. Nature 318, 533-538 (1985).

39. Land, H., Parada, L. F. \& Weinberg, R. A. Tumorigenic conversion of primary embryo fibroblasts requires at least two cooperating oncogenes. Nature 304, 596-602 (1983).

40. Bissonnette, R. P., Echeverri, F., Mahboubi, A. \& Green, D. R. Apoptotic cell death induced by c-myc is inhibited by bcl-2. Nature 359, 552-554 (1992).

41. Shi, $Y$. et al. Role for c-myc in activation-induced apoptotic cell death in T cell hybridomas. Science 257, 212-214 (1992).

42. Kocak, $\mathrm{H}$. et al. Hox-C9 activates the intrinsic pathway of apoptosis and is associated with spontaneous regression in neuroblastoma. Cell Death Dis. 4, e586 (2013).

43. Zhang, W. et al. Comparison of RNA-seq and microarray-based models for clinical endpoint prediction. Genome Biol. 16, 133 (2015).

44. Riemer, J., Hoepken, H. H., Czerwinska, H., Robinson, S. R. \& Dringen, R. Colorimetric ferrozine-based assay for the quantitation of iron in cultured cells. Anal. Biochem. 331, 370-375 (2004). 